\title{
Comparative genomic analysis reveals a novel mitochondrial isoform of human rTS protein and unusual phylogenetic distribution of the rTS gene
}

\author{
Ping Liang*1, Jayakumar R Nair ${ }^{2}$, Lei Song1, John J McGuire ${ }^{2}$ and \\ Bruce J Dolnick*2
}

Address: ${ }^{1}$ Department of Cancer Genetics, Roswell Park Cancer Institute, Buffalo, USA and ${ }^{2}$ Department of Pharmacology and Therapeutics, Roswell Park Cancer Institute, Buffalo, USA

Email: Ping Liang* - Ping.Liang@roswellpark.org; Jayakumar R Nair - R.Jayakumar@ RoswellPark.org; Lei Song - Lei.Song@RoswellPark.org; John J McGuire - John.McGuire@RoswellPark.edu; Bruce J Dolnick* - Bruce.Dolnick@RoswellPark.org

* Corresponding authors

Published: 14 September 2005

BMC Genomics 2005, 6:125 doi:10.1 186/147|-2164-6-125

This article is available from: http://www.biomedcentral.com//47|-2/64/6//25

(c) 2005 Liang et al; licensee BioMed Central Ltd.

This is an Open Access article distributed under the terms of the Creative Commons Attribution License (http://creativecommons.org/licenses/by/2.0), which permits unrestricted use, distribution, and reproduction in any medium, provided the original work is properly cited.

\begin{abstract}
Background: The rTS gene (ENOSFI), first identified in Homo sapiens as a gene complementary to the thymidylate synthase (TYMS) mRNA, is known to encode two protein isoforms, $r T S \alpha$ and rTS $\beta$. The $r$ TS $\beta$ isoform appears to be an enzyme responsible for the synthesis of signaling molecules involved in the down-regulation of thymidylate synthase, but the exact cellular functions of rTS genes are largely unknown.
\end{abstract}

Results: Through comparative genomic sequence analysis, we predicted the existence of a novel protein isoform, $r T S$, which has a 27 residue longer $\mathrm{N}$-terminus by virtue of utilizing an alternative start codon located upstream of the start codon in rTS $\beta$. We observed that a similar extended $\mathrm{N}$ terminus could be predicted in all rTS genes for which genomic sequences are available and the extended regions are conserved from bacteria to human. Therefore, we reasoned that the protein with the extended $\mathrm{N}$-terminus might represent an ancestral form of the rTS protein. Sequence analysis strongly predicts a mitochondrial signal sequence in the extended $\mathrm{N}$-terminal of human rTS $\gamma$, which is absent in rTS $\beta$. We confirmed the existence of $r T S$ in human mitochondria experimentally by demonstrating the presence of both rTS $\gamma$ and $r T S \beta$ proteins in mitochondria isolated by subcellular fractionation. In addition, our comprehensive analysis of $r$ TS orthologous sequences reveals an unusual phylogenetic distribution of this gene, which suggests the occurrence of one or more horizontal gene transfer events.

Conclusion: The presence of two rTS isoforms in mitochondria suggests that the rTS signaling pathway may be active within mitochondria. Our report also presents an example of identifying novel protein isoforms and for improving gene annotation through comparative genomic analysis.

\section{Background}

The rTS (ENOSF1) gene, a member of the enolase family, was initially identified in Homo sapiens by the discovery of an RNA with extensive complementarity to the mRNA for the DNA biosynthetic enzyme thymidylate synthase $[1,2]$. The rTS gene was later shown to code for two proteins 
(rTS $\alpha$ and $\mathrm{rTS} \beta$ ) through alternative RNA splicing $[2,3]$. The mRNA for rTS $\alpha$ is complementary to thymidylate synthase mRNA, while the mRNA for rTS $\beta$ is not $[2,3]$. The rTS $\beta$ protein is the major protein product of the $r$ TS gene and its expression is associated with the down-regulation of thymidylate synthase protein as cultured cells enter growth arrest [2]. Expression of rTS $\beta$ correlates with the production of small molecules that appear to mediate the down-regulation of thymidylate synthase protein by a novel intercellular signaling mechanism [2]. Overproduction of rTS $\beta$ occurs in some cells resistant to inhibitors of thymidylate synthase or dihydrofolate reductase, indicating a role for the $r T S$ gene in folate and nucleotide metabolism, as well as anticancer drug resistance [2-6].

While the specific function(s) of the rTS gene products are currently under investigation, we now report a new rTS protein isoform and its association with mitochondria. The existence of this new isoform, rTS $\gamma$, was first predicted using a computational comparative genomic sequence analysis approach and was then verified experimentally. This unexpected observation suggests that rTS may have functions in addition to intercellular signaling.

\section{Results \\ A conserved extended protein $\mathbf{N}$-terminus can be deduced from all available $\mathrm{rTS}$ genes}

Comprehensive analysis of all available database sequences revealed that $r$ TS genes demonstrate an atypical phylogenetic distribution. $r$ TS exists only in a few groups of eubacteria, two fungal lineages (Ascomycota and Basidiomycota), and most animal species from insects to mammals. Among bacterial rTS orthologous genes, several were annotated with a longer $\mathrm{N}$-terminus based on a start codon located further upstream. These proteins include NP_355739.1 (Agrobacterium tumefaciens str. C58), NP_540624.1(Brucella melitensis 16M), NP_639408.1 (Xanthomonas campestris pv. campestris str. ATCC 33913), NP_669902.1 (Yersinia pestis KIM), NP_828458.1 (Streptomyces avermitilis MA-4680), CAD61030.1 (Arthrobacter ilicis), and ZP_00227861.1 (Kineococcus radiotolerans SRS30216), while many other proteins, including NP_405150.1 (Yersinia pestis CO92), NP_437232.1 (Sinorhizobium meliloti), NP_533476.1 (Agrobacterium tumefaciens str. C58), NP_744975.1 (Pseudomonas putida KT2440), ZP_00213853.1 (Burkholderia cepacia R18194), ZP_00281771.1 (Burkholderia fungorum LB400), AAM39023.1 (Xanthomonas axonopodis pv. citri str. 306), and YP_070105 (Yersinia pseudotuberculosis) were annotated with an $\mathrm{N}$-terminus equivalent to that of human rTS $\beta$. Therefore, we determined whether an equivalent extended N-terminus could be predicted in the human $r T S$ gene. Previously, all available human $r T S$ genomic sequences appeared to contain a sequence gap immediately upstream of the start codon of rTS $\beta$, and the pub- lished 5'-end of the rTS mRNAs was originally determined by RACE (Rapid Amplification of cDNA Ends) analysis of cloned sequences [2]. Thus, a longer $\mathrm{N}$-terminal was not predicted initially, and not expected based upon the existing experimental evidence. However, at the time we started this analysis, one BAC clone, RP11-778P8 [AC021474.1], was found to contain the sequence covering an extended exon region as well as the rest of the gene, although its sequence was in a status of unordered fragments. In addition, a GenBank entry for the human $r$ TS gene by Dolnick and Su [AF305057] contains the complete 5 -end upstream sequence. Analysis of this sequence by GenScan [7] predicts a start codon upstream of the start codon for rTS $\beta$, yielding an extended $\mathrm{N}$-terminal that is 27 amino acids longer than rTS $\beta$ (Fig. 1A). A sequence comparison between the extended human rTS protein region and the bacterial rTS proteins possessing a longer $\mathrm{N}$-terminus revealed a high level of sequence similarity in the extended region. Therefore, we reasoned that a longer $\mathrm{N}$ terminus may exist in all $r$ TS orthologous genes, and this was confirmed by the presence of a possible equivalent extension of the N-terminus in all available $r T S$ genomic sequences (Fig. 1A-E). We named this potential new isoform rTS $\gamma$ to distinguish it from rTS $\beta$ and rTS $\alpha$. During our preparation of this manuscript, NCBI released a new RefGene entry for human rTS $\beta$ [NM_202758 and NP_059982.1]. This entry, dated Dec-20-2004, predicted a different $\mathrm{N}$-terminus and was subsequently replaced by another entry [NM_017512 and NP_059982.2] on 02March-2005, which has a deduced protein product identical to that described in this report. While all available animal $r T S$ genes, including those from human, three fish species (fresh and salt water pufferfish and zebrafish), the basal chordate, Ciona intestinalis, and the invertebrate Anopheles gambiae, share the same intron/exon boundary position at least for the first exon/intron junction (Fig. $1 \mathrm{~A} \& \mathrm{~B})$, the fungal $r T S$ genes seem to vary in gene structure making them different from the animal genes, as well as from each other (Fig. 1C\&D).

In addition to the genomic sequences, EST sequences for $r T S$ genes containing sufficient 5 '-end sequences were also identified for a few more animal species, including cow, rat, frog, multiple bony fish species, sea squirt, beetles and mites. Multiple sequence alignment analysis revealed that the extended N-termini of $r T S$ genes are conserved from bacteria to human (Fig. 2). Therefore, we believe that the extended $\mathrm{N}$-terminal region represents an ancestral form of the $r T S$ gene products.

\section{The extended $\mathbf{N}$-terminus contributes a mitochondrial signal in human $r$ TS $\gamma$ protein}

During our search for potential new motifs and/or functions contributed by the extended $\mathrm{N}$-terminal region of rTS, we found that this extended sequence was predicted 
A. Homo sapiens (Human)

tcccgaccagtcctgaccgcacgggggecgcggccacggggcgcaggggccatggtgcgc M V R ggcaggatctcccggctctcggtccgggacgtgcgcttccccacgtcgcttgggggccac $\begin{array}{llllllllllllllllllll}G & R & I & S & R & L & S & V & R & D & V & R & F & P & T & S & L & G & G & H\end{array}$ ggcgcggacgccatggtaagcg..(6kb intron)..ctcctgcagcacacggaccct. . G A D A $\mathrm{M}$

B. Anopheles gambiae (mosquito)

ctcagcagcatggggaaggatcggtgcctcaacattaccaccctgcaggcaaaggatatc

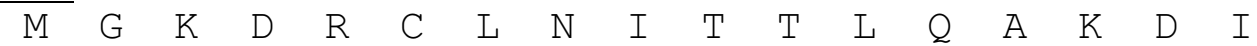
Cgatggccaacgtcactgggagcgcacgggtccgatgcgatggtatgtgtgcgtcgaa. . $\begin{array}{llllllllllllll}R & W & P & T & S & \text { L } & G & A & H & G & S & D & A & M\end{array}$ ..(76 bp intron)..tccgatcaaagtagcacacggatccggactattcctgcgt.. $\begin{array}{llllllllllllll}\mathrm{H} & \mathrm{T} & \mathrm{D} & \mathrm{P} & \mathrm{D} & \mathrm{Y} & \mathrm{S} & \mathrm{C} & \mathrm{V} & \end{array}$

C. Ustilago maydis (fungi, Basidiomycetes)

ttgattgacatcaaattactaagtaccgccttgcctaccatgaccaagttatcgccgctg M T K L $\mathrm{S}$ P L gtcgtctcgtcgtattcgatacacgatgtacgctttcccacttcgctcaccggagatggc $\begin{array}{llllllllllllllllllll}\mathrm{V} & \mathrm{V} & \mathrm{S} & \mathrm{S} & \mathrm{Y} & \mathrm{S} & \mathrm{I} & \mathrm{H} & \mathrm{D} & \mathrm{V} & \mathrm{R} & \mathrm{F} & \mathrm{P} & \mathrm{T} & \mathrm{S} & \mathrm{L} & \mathrm{T} & \mathrm{G} & \mathrm{D} & \mathrm{G}\end{array}$ Acggacgctatgaacaagtgtaagtgtagtaagtgtacaagccaccgttaatgagcag . • $\mathrm{T} \quad \mathrm{D} \quad \mathrm{A} \overline{\mathrm{M}} \mathrm{N} \quad \mathrm{K}$

D. Magnaporthe grisea (fungi, Ascomycetes) ttctctgtccagctgtttatcctttgacttattttcttataatcttctggtcaaaata atggcagctcaagaaatcaccattacgggctggaaaacccgcgatgtccggttccccaca $\begin{array}{lllllllllllllllllllll}M & A & A & Q & E & I & T & I & T & G & W & K & T & R & D & V & R & F & P & T\end{array}$ tctttggacaagacggggtcggatgccatgaacgctgcgggtgattactcgtcagcctat $\begin{array}{llllllllllllllllllll}S & L & D & K & T & G & S & D & A & M & N & A & A & G & D & Y & S & S & A & Y\end{array}$ tgcatccttgagacagactcagagttctcaggtcatggaatggttagtagtggcatatat $\begin{array}{llllllllllllllllllllll}C & \mathrm{I} & \mathrm{E} & \mathrm{T} & \mathrm{D} & \mathrm{S} & \mathrm{E} & \mathrm{F} & \mathrm{S} & \mathrm{G} & \mathrm{H} & \mathrm{G} & \mathrm{M}\end{array}$

E. Sinorhizobium meliloti ( $\alpha$-proteobacteria) cgctgcaggtctattggagcaaacatgacccgcatcaccgaccttcgcgtcttcgacctt $\begin{array}{lllllllllllll}\mathrm{M} & \mathrm{T} & \mathrm{R} & \mathrm{I} & \mathrm{T} & \mathrm{D} & \mathrm{L} & \mathrm{R} & \mathrm{V} & \mathrm{F} & \mathrm{D} & \mathrm{L}\end{array}$ cgctttccgacctcggcgagcctcgacggttcggacgccatgaacccggatccggactat

$\begin{array}{llllllllllllllllllllll}R & \mathrm{~F} & \mathrm{P} & \mathrm{T} & \mathrm{S} & \mathrm{A} & \mathrm{S} & \mathrm{L} & \mathrm{D} & \mathrm{G} & \mathrm{S} & \mathrm{D} & \mathrm{A} & \mathrm{M} & \mathrm{N} & \mathrm{P} & \mathrm{D} & \mathrm{P} & \mathrm{D} & \mathrm{Y}\end{array}$

\section{Figure I}

Prediction of extended $\mathbf{N}$-termini for $\mathbf{r T S}$ genes. The genomic sequences and the predicted protein translations for the $\mathrm{N}$-terminal regions from four different species, Homo sapiens, Anopheles gambiae, Ustilago maydis, and Sinorhizobium meliloti, are shown in panels A to D, respectively. A double underlined "ATG" indicates the predicted start codon for the extended N-terminus, while the "ATG" with single underline indicates the start codon of the isoform with a shorter N-terminus. Grey highlights indicate the canonical "GT....AG" intron motif. 


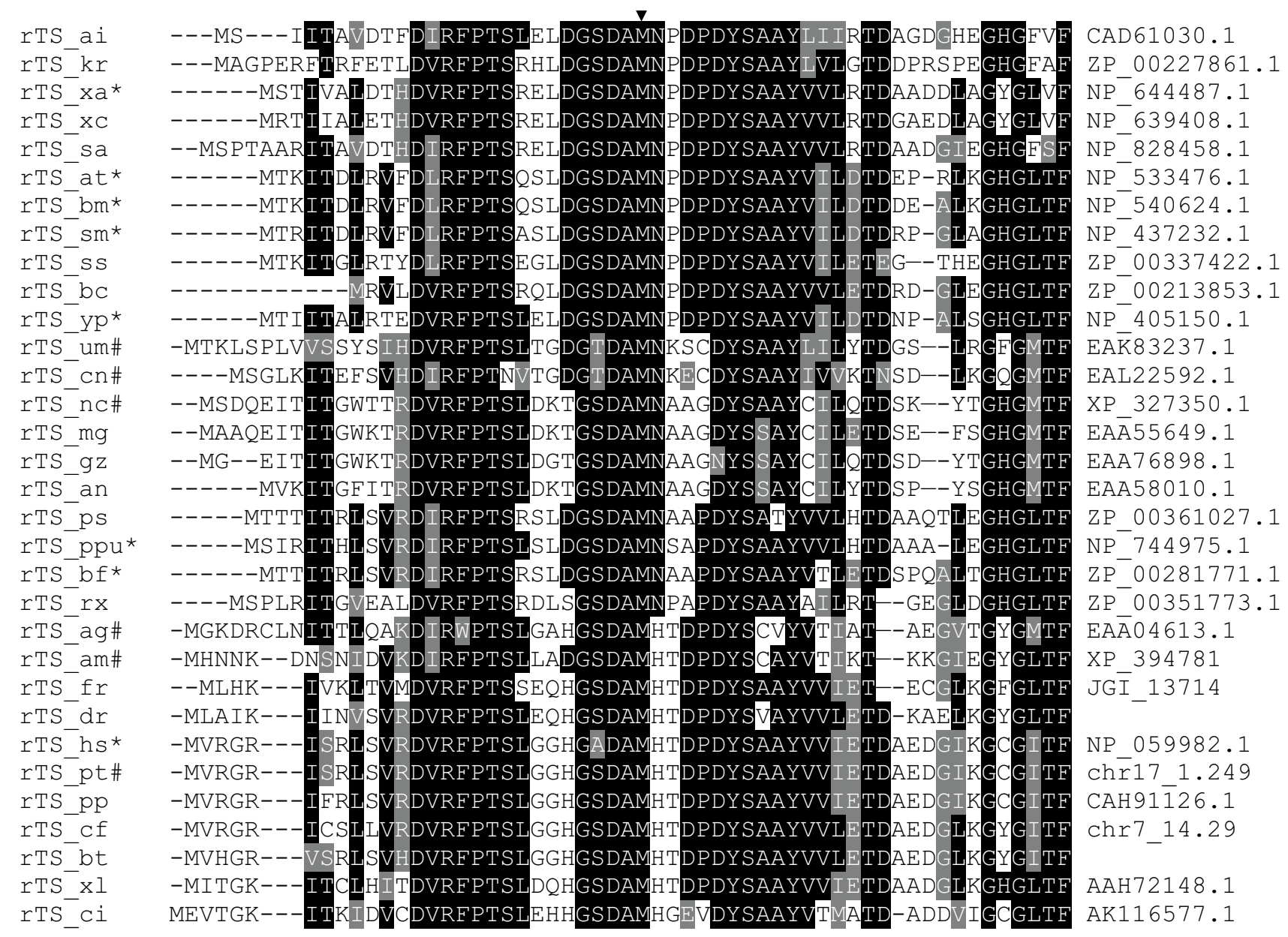

\section{Figure 2}

Sequence conservation within the extended $\mathbf{N}$-terminal regions among all available $\mathbf{r T S}$ genes. The $\mathrm{N}$-terminal section of all available $r T S$ protein sequences were analyzed using Clustal $X$ followed by boxshading using the BOXSHADE server [32]. The arrow indicates the start methionine in the short form of rTS proteins. Black boxed residues are identical, grey boxed residues are similar. The species name of each sequence is indicated by the last two letters in the sequence ID (ag, Anopheles gambiae; ai, Arthrobacter ilicis; am, Apis mellifera; an, Aspergillus nidulans; at, Agrobacterium tumefaciens; bc, Burkholderia cepacia; bf, Burkholderia fungorum; bm, Brucella melitensis; bt, Bos tauras; $c f$, Canis familiaris; ci, Ciona intestinalis; cn, Cryptococcus neoformans; dr, Danio rerio; fr, Takifugu rubripes; gz, Gibberella zeae; hs, Homo sapien; kr, Kineococcus radiotolerans; mg, Magnaporthe grisea; nc, Neurospora crassa; pp, Pongo pygmaeus; ppu, Pseudomonas putida KT2440; ps, Polaromonas sp. JS666; pt, Pan troglodytes; rx, Rubrobacter xylanophilus; sa, Streptomyces avermitilis; sm, Sinorhizobium meliloti; ss, Silicibacter sp. TMI040; tr, Takifugu radiatus; um, Ustilago maydis; $x a$, Xanthomonas axonopodis; $x c$, Xanthomonas campestris; $x l$, Xenopus laevis; $y p$, Yersinia pestis). The accession numbers of the sequences are provide in the last column of the alignment. Sequences labeled with $*$ are those annotated with a shorter $\mathrm{N}$-terminus, while those labeled with \# are the ones containing extra sequences at the $\mathrm{N}$-terminus (see complete sequences in Additional file I).

to contain a mitochondrial signal. As shown in Table 1, all available programs predict a strong mitochondrial signal for rTS $\gamma$, but not for rTS $\beta$, suggesting that the mitochondrial signal is conferred by the extended N-terminal sequence.

\section{rTS $\gamma$ protein exists and is associated with mitochondria in} human cells

We addressed the existence of the rTS $\gamma$ isoform and its possible association with mitochondria experimentally. Initially, a cytosolic fraction and an organellar pellet 
Table I: Predicted mitochondrial signal for $\mathrm{rTS} \beta$ and $\gamma$ proteins

\begin{tabular}{llll}
\hline Program & $r T S \gamma$ & $r T S \beta$ & Reference \\
\hline Mitoprot & 0.9382 & 0.0117 & {$[22]$} \\
TargetP & 0.719 & 0.077 & {$[23]$} \\
PSORTII & $26.1 \%$ & $8.7 \%$ & {$[24]$} \\
MITOPRED & $92.3 \%$ & 0 & {$[25,26]$} \\
\hline
\end{tabular}

(including mitochondria and lysosomes) were prepared from CCRF-CEM human cells, and their proteins were resolved by electrophoresis and analyzed for rTS protein expression (Fig. 3A). The results indicate that two rTS proteins with apparent molecular mass of $52.7 \pm 1.8$ and 47.6 $\pm 0.7 \mathrm{kDa}$ (approximately corresponding to the difference in the predicted molecular mass of the rTS $\beta$ and $\gamma$ isoforms, respectively) are present. There was a preferential distribution of the higher molecular mass species in the organellar fraction as compared to the cytosolic fraction. The presence in the organellar fraction of both lysosomal (LAMP-1) and mitochondrial (MnSOD) marker proteins, however, did not allow us to conclude that rTS proteins are present in the mitochondria, rather than in the lysosomes. To resolve this, we partially separated lysosomes from mitochondria using a $5-20 \%$ iodixanol gradient $[8,9]$ (Fig. 3B). The protein profile of the iodixanol gradient shows a peak centered on fractions $14-15$ with a pronounced shoulder in fractions 10-12. GDH activity appears in fractions 12-18 with a peak at fraction 15, indicating the presence of mitochondria in these fractions. The shoulder in the protein profile (fractions 10-12) that lacks GDH activity suggests the presence of non-mitochondrial organelles, including lysosomes. This suggestion was confirmed by Western blotting of the gradient fractions for MnSOD and LAMP-1 markers (Fig. 3C). Analysis of the distribution of rTS $\beta$ in the gradient indicates that both the rTS $\beta$ and $\gamma$ species co-localize with MnSOD, but not LAMP-1, conclusively demonstrating their presence in mitochondria, but not lysosomes.

\section{Discussion \\ Functional implications for the rTS gene based on its mitochondrial location}

It has recently been determined that rTS $\beta$ is an enzyme responsible for the synthesis of small signaling molecules involved with the down-regulation of thymidylate synthase as cells enter growth arrest in vitro [6]. The signaling associated with rTS $\beta$ was also shown to act intercellularly [6]. Sequence analysis of the rTS gene suggested the presence of a mitochondrial leader sequence that would be expected to increase the length of rTS $\beta$ from 416 to 443 amino acids in rTS $\gamma$ with an expected change in molecular mass from 46,892 to 49,742 Da. Based upon the predic- tion of a mitochondrial leader sequence, we evaluated whether there is a mitochondrial form of the rTS protein. The results shown in Fig. 3 indicate that this is the case, with two species being present in the mitochondria. These two protein species have apparent molecular masses of $52.7 \pm 1.8$ and $47.6 \pm 0.7 \mathrm{kDa}$. The smaller species corresponds well to the predicted size expected for rTS $\beta$ with the mitochondrial leader sequence cleaved off, while the larger species differs by $3 \mathrm{kDa}$ from the predicted molecular mass for the isoform with the leader sequence. The amounts of rTS $\gamma$ and rTS $\beta$ in the combined cytoplasmic and mitochondrial fractions differ from that expected to result from combining equal amounts of each fraction. The increased abundance of rTS $\beta$, relative to rTS $\gamma$ in the combined fraction may be the result of in situ cleavage of the rTS $\gamma$ mitochondrial leader sequence by cytosolic proteases, which has been observed to occur in yeast cytosol [10], although transfer of protein may also contribute to the change in signal strength as the MnSOD signal is weaker in the lane with the combined fractions than in the lane loaded with just the organelle protein. Although the migration of proteins in SDS-PAGE gives only approximate estimation of molecular masses, there is a possibility that other post-translational modifications may contribute to this discrepancy. The co-localization of rTS $\beta$ and rTS $\gamma$ with GDH and MnSOD after subcellular fractionation strongly suggests that the extended $\mathrm{N}$-terminal sequence serves as the leader sequence for mitochondrial location of the protein and that it is likely this is partially cleaved to generate the $\beta$ isoform, once rTS $\gamma$ is transported into the mitochondria. The presence of the rTS $\beta$ protein in mitochondria raises the question of what role this enzyme and the rTS signaling pathway may play there. Mitochondria are a major site for folate metabolism in mammalian cells [11]. Thymidylate synthase is also found within mitochondria [12], despite the absence of a canonical mitochondrial leader sequence, and the relationship of rTS signaling to thymidylate synthase and folate metabolism may ultimately provide the explanation for this phenomenon. Recent evidence indicates that treatment of cells with an rTS $\beta$ signaling mimic can affect the cytoskeleton and cause down-regulation of TYMS $[10,13]$. The colocalization of rTS and thymidylate synthase in the mitochondria may indicate that rTS $\beta$, in addition to its role in intercellular signaling, also provides intracellular signals that regulate thymidylate synthase levels in the mitochondria.

Since the extended N-termini of rTS are conserved from bacteria to human, we believe that the rTS $\gamma$ form of the $r T S$ gene represents the ancestral form of this gene, while rTS $\beta$, which seems to be the predominantly expressed form in the cytosol, represents an isoform that appeared later during evolution and came to co-exist with the ancestral isoform, at least in Homo sapiens. Based on a recent study 

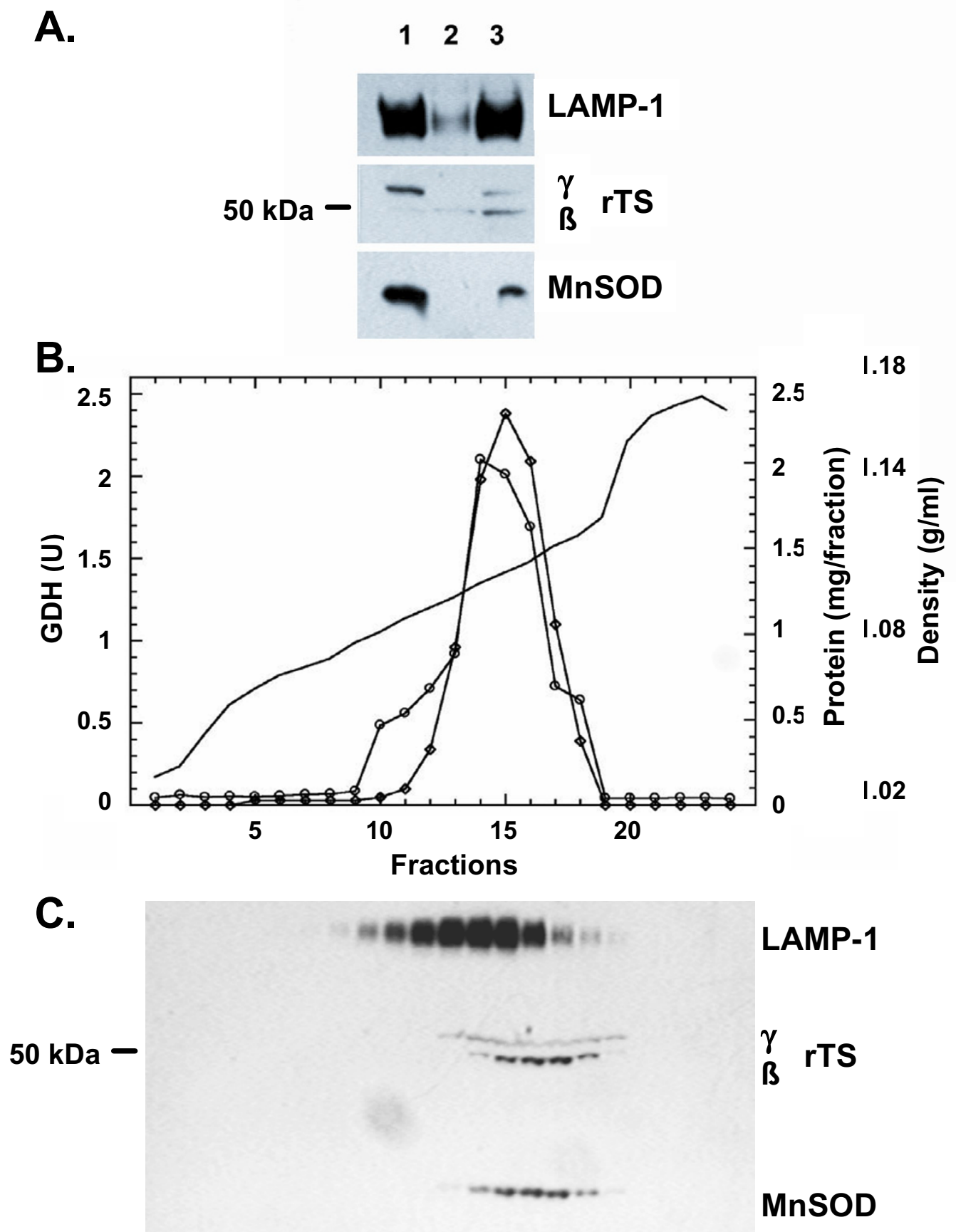

Figure 3

Localization of rTS $\beta$ and rTS $\gamma$ to mitochondria. A. Presence of rTS $\beta$ in both cytosol and organelle fractions. Five $\mu \mathrm{g}$ of protein from the organelle (lane I), cytosol (lane 2), or $5 \mu \mathrm{g}$ each of the organelle plus cytosol (lane 3) were resolved by gel electrophoresis, blotted and probed for the presence of the indicated proteins. B. Gradient fractionation of mitochondria and lysosomes. The crude organellar suspension from CCRF-CEM cells was fractionated on a linear iodixanol gradient as described. Individual fractions were collected and assayed for GDH activity and protein content. -, Density ( $g / m l)$ of fractions from a mock 5-20\% iodixanol gradient; - $\diamond-$, GDH activity; -O-, Protein content. C. Western blot. Five $\mu$ l of each fraction was denatured and processed for Western blotting as described. 


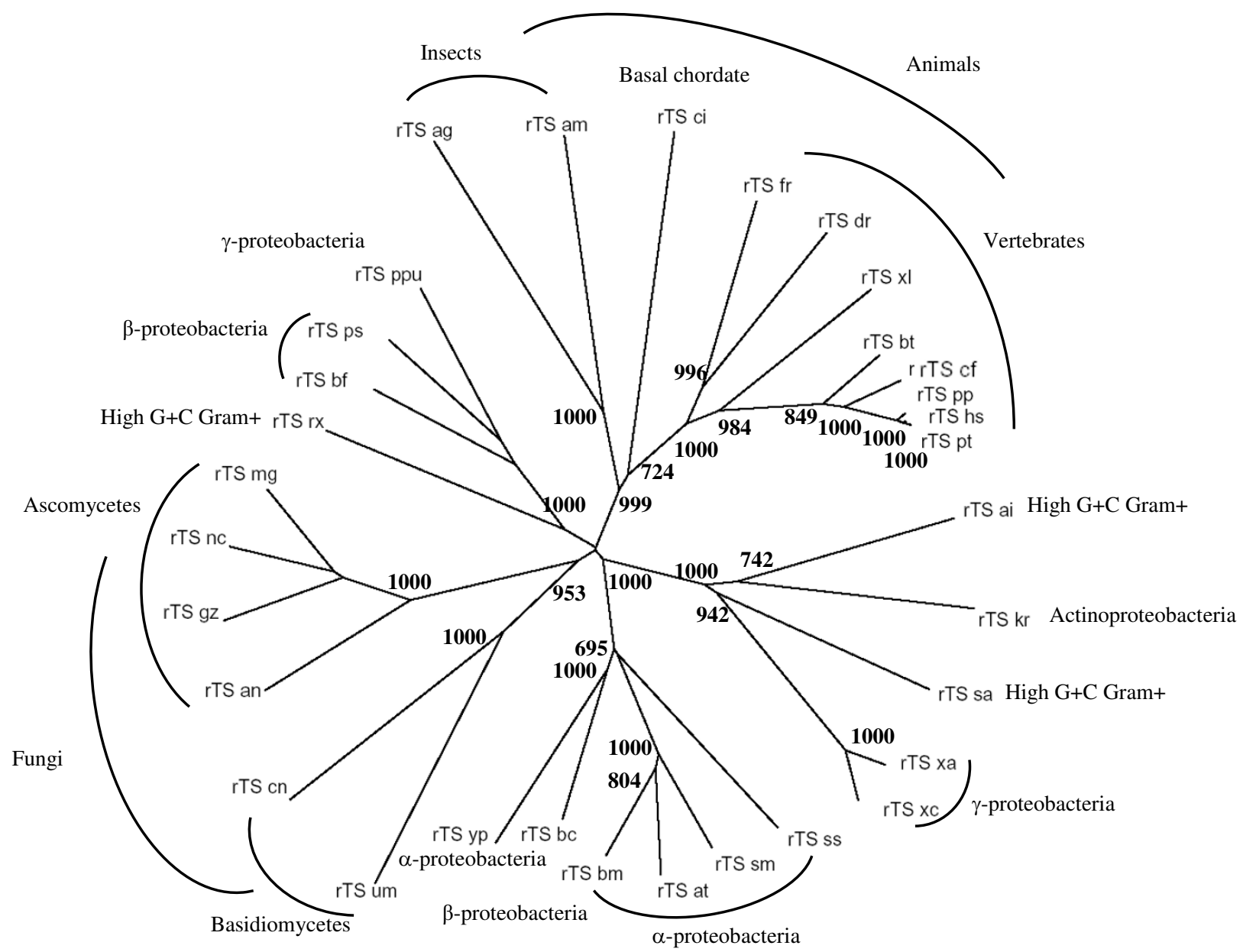

\section{Figure 4}

Unusual phylogenetic distribution of the rTS gene. The sequences of the same set of proteins displayed in Fig. 2 were used to generate a neighbor-joining tree using ClustalX. The tree was displayed in TreeView program, while the taxonomic labels of the species were added manually afterward. In addition to the extension of the $\mathrm{N}$-terminus as indicated in Fig. 2 , adjustments to the currently annotated exon boundaries for a few rTS genes were necessarily made based on the best match between their genomic sequences and the human $r T S \gamma$ protein sequence (see detailed modifications in supplemental materials). Bootstrap values that are over 700 (1000 trials) are shown at the nodes.

which shows that suboptimal AUG codons can support translation via leaky scanning and reinitiation [14], the co-existence of protein products translated using different AUG codons in the same reading frame may not be a rare phenomena. In fact, the starting AUG codons for rTS $\gamma$ and rTS $\beta$ are both qualified as optimal start codons according to recent studies of translation initiation in mammalian and plant genes [14-16], providing an explanation for the co-existence of rTS $\gamma$ and rTS $\beta$. A recent study suggests that many alternative splicing forms cause differential subcellular localization, especially in targeting either peroxisomes or mitochondria [17], and our data serves as evidence supporting such a notion. An interesting subject for future studies will be to determine when the shorter rTS $\beta$ isoform appeared during evolution.

\section{Phylogenetic distribution and origin of rTS gene}

rTS is highly conserved, being found in a variety of species, ranging from bacteria to human. Unlike other enolase genes with a wider phylogenetic distribution, or the thymidylate synthase (TYMS) gene, which is ubiquitous in organisms from all three kingdoms and is highly conserved, $r T S$ demonstrates an unusual phylogenetic distribution. As shown in Fig. 4, the presence of $r T S$ is limited 
to a few groups of eubacteria including $\alpha_{-}, \beta_{-}$, and $\gamma$-proteobacteria, actinobacteria, some fungi, and animal species spanning the phylogenetic range from insects to mammals. Among the vertebrate species for which draft genome sequences and/or a large number of EST sequences are available, we were able to identify rTS sequences from all species with the exception of chicken and mouse [see a complete list of deduced amino acid sequences in Additional file 1]. Furthermore, we observed in all vertebrate species with sufficient genome sequences including human, chimpanzee, rat, and fugu fish, that the TYMS-rTS gene pair is part of a large conserved gene synteny among vertebrates (data not shown). While the absence of $r T S$ sequence in chicken may be due to the insufficiency of available genome sequences or ESTs, we were puzzled by the failure to retrieve any $r T S$ sequences in mouse, considering the fact that the mouse genome sequence is now fairly complete and its EST data is quite comprehensive. However, we recently obtained preliminary data showing expression of mouse rTS protein and mRNA (data not shown). Another unusual observation is that the rTS gene does not seem to be present in E. coli while the rTS gene is present in another enterobacterium,Yersinia pestis. Similarly, although the rTS gene is present in Anopheles gambiae and several other insect species, but is not found in Drosophila. So far, no rTS gene has been identified in any plant or yeast species despite the fact that complete genome sequences and comprehensive EST sequences are available for a number of species in these lineages. We also find no rTS sequences from Caenorhabditis elegans or other worm genome sequences. The same is true for all archaebacteria and many eubacteria lineages. These observations suggest that the rTS gene originated as a bacterial gene which was either horizontally transferred into certain animal and fungal lineages, or alternatively, was lost in all the lineages that do not contain rTS genes. An interesting observation is that, while the rTS branches for the animal lineage and the fungal lineage show a topology that agrees well with commonly accepted tree of life for these species, the branches for the bacterial lineages conflict with the commonly accepted tree for the bacterial lineages (Fig. 4). The latter is demonstrated by the fact that species from the same lineage do not always group together, while species from different bacterial lineages do cluster together in many cases (Fig. 4). For example, one $\beta$-proteobactial rTS sequence (rTS_bc) is grouped with $\alpha$-proteobacterial sequences, while the other two $\beta$-proteobacterial sequences cluster with a $\gamma$-proteobacterial sequence and the three rTS sequences from the High $\mathrm{G}+\mathrm{C}$ gram positive bacteria are located on three different branches, which all consist of sequences from multiple groups (Fig. 4A). Although not all nodes for the bacterial groups are supported by a high bootstrap value, many nodes are supported with high confidence. This type of unexpected phylotree shown by the rTS sequences suggests the possibility of horizontal gene transfer among the bacteria species, which is common [19-21]. The fact that most of the bacteria and fungi that have a $r T S$ gene are human and/or animal pathogens (Aspergillus nidulans, Brucella suis, Brucella melitensis, Burkholderia cepacia Burkholderia fungorum, Cryptococcus neoformans, Yersinia pestis, Silicibacter sp), plant pathogens (Agrobacterium tumefaciens, Gibberella zeae, Xanthomonas axonopodis, Xanthomonas campestris., Ustilago maydis, Magnaporthe grisea), or in one instance a plant symbiont ( $S$. meliloti), adds weight to the hypothesis of horizontal gene transfer events. In the case of plant pathogens, the plants can serve as a mediator between a bacterial donor and an animal acceptor. This may also suggest that the rTS gene is required to create and/or maintain a certain type of hostpathogen relationship. Therefore, a better understanding of the biological function of the rTS gene may provide new insights for disease control related to these bacterial pathogens.

In addition to the discovery of this novel mitochondrial isoform of rTS, our report also represents a good illustration of the use of comparative genomic analysis for identifying novel protein isoforms of genes and for improving existing gene predictions. Through this study, we have made a comprehensive collection of rTS orthologous sequences and have made a list of suggested modifications to the existing gene annotation (see detailed modifications in the supplemental materials). In addition, by searching all annotated human genes, we identified a few other genes in which evolutionarily conserved alternative upstream AUG codons exist (similar situation as rTS $\beta$ ). These genes include SELL [NM_000655], SPCS1 [NM_014041.1], NT5C3 [NM_016489], and SDN1 [NM_014390] (data not shown).

\section{Conclusion}

In summary, through comparative genomic analyses we revealed an unusual phylogenetic distribution of the $r T S$ gene and identified a novel mitochondria isoform of this gene and verified it experimentally. A mitochondrial location of rTS protein and phylogenetic distribution of this gene provide us with new information that will assist in elucidating its function.

\section{Methods \\ Identification of an extended $\mathbf{N}$-terminal region for all available rTS genes}

To search for all available rTS orthologous sequences, we first queried all protein sequences deposited in the NCBI non-redundant protein database (nr) by BLAST search [21]. Among all identified rTS $\beta$ orthologous protein sequences, the ones with an $\mathrm{N}$-terminus equivalent to human rTS $\beta$ were identified and their corresponding genomic sequences, including sufficient 5 '-end upstream 
sequences for analysis, were retrieved. Extended N-termini were deduced by extending the start codon to the next available "ATG" upstream of the start codon used by the existing annotation. In addition to the rTS orthologous genomic sequences, we also searched the NCBI EST database by performing a TBLASTN search with the extended human rTS $\beta$ protein sequence to collect rTS cDNA sequences from additional species. To predict any potential new function or cellular location contributed by the extended N-terminus, we analyzed the human rTS $\gamma$ and rTS $\beta$ protein sequences with Mitoprot [22], TargetP [23], PSORTII [24], and Mitopredict [25,26].

\section{Subcellular fractionation of CCRF-CEM cells}

The human T-lymphoblastic cell line CCRF-CEM was cultured as described [27]. Subcellular fractions of CCRFCEM cells were isolated essentially as described [28], except that protease ( $0.5 \mathrm{mM}$ Pefabloc) and phosphatase inhibitors ( $1 \mathrm{mM}$ sodium metavanadate and $1 \mathrm{mM} \mathrm{NaF}$ ) were included in all buffers. All steps were performed at 0 $-4^{\circ} \mathrm{C}$. Briefly, 1-liter of CCRF-CEM cells ( $\sim 3.5 \times 10^{5}$ cells/ $\mathrm{ml}$ ) was centrifuged at $1000 \times \mathrm{g}$ for $5 \mathrm{~min}$ and the pellet was washed with iced $0.9 \% \mathrm{NaCl}$, and then suspended in 5 pellet volumes of ice-cold hypotonic buffer [30] and allowed to swell for $5 \mathrm{~min}$ on ice. The suspension was homogenized in an ice-cold 7-ml glass Dounce homogenizer with 15 strokes of the tight pestle to obtain > 95\% cell disruption. The homogenate was immediately made up to $250 \mathrm{mM}$ sucrose and centrifuged at $1000 \times \mathrm{g}$ for 5 min. The pellet was washed once with 2.5 original pellet volumes of cold isotonic buffer $\left(1 \mathrm{mM} \mathrm{Na}_{2}\right.$-EDTA, 250 $\mathrm{mM}$ sucrose; $\mathrm{pH}$ 6.9). The two supernatants were combined to generate the post-nuclear supernatant (PNS). The PNS was centrifuged at $17,000 \times \mathrm{g}, 15 \mathrm{~min}$ to generate a cytosolic fraction (supernatant) and an organellar pellet containing both mitochondria and lysosomes. The organellar pellet was suspended in $1 \mathrm{ml}$ of HES $(20 \mathrm{mM}$ HEPES-NaOH, $1 \mathrm{mM} \mathrm{Na}$-EDTA, $250 \mathrm{mM}$ sucrose; $\mathrm{pH}$ 7.4). Subcellular fractions were assayed in duplicate for activity of the cytosolic enzyme lactate dehydrogenase (LDH) [30] and mitochondrial matrix enzyme glutamate dehydrogenase (GDH) [31] to ensure that fractionation was successful. To separate mitochondria and lysosomes, this organellar suspension was made up to $30 \%(\mathrm{w} / \mathrm{v})$ iodixanol $[8,32]$ in a final volume of $2.2 \mathrm{ml}$ and placed on a $5-20 \%$ linear iodixanol gradient $(9 \mathrm{ml})$, centrifuged at $70,000 \times \mathrm{g}$ for $1.5 \mathrm{hr}$, and collected as $0.5-\mathrm{ml}$ fractions, which were then diluted with $0.5 \mathrm{ml}$ ice-cold HES and centrifuged at $30,000 \times \mathrm{g}$ for $15 \mathrm{~min}$. The resulting organelle pellets were suspended in $200 \mu \mathrm{l}$ of isotonic buffer (as above, except that $\mathrm{NaF}$ was $50 \mathrm{mM}$ ). Protein concentration was determined using the BioRad (Hercules, CA) protein assay kit.

\section{Western blotting}

Proteins were resolved by denaturing gel electrophoresis using $10 \%$ polyacrylamide and transferred to PVDF membranes essentially as described [4]. The primary antibodies used were: D3 (mouse monoclonal to rTS $\beta$ ), LAMP-1 (mouse monoclonal to lysosome-associated membrane protein-1; Santa Cruz Biotechnology), and MnSOD (rabbit polyclonal to manganese superoxide dismutase; Stressgen). Secondary antibodies consisted of horseradish peroxidase conjugated $\mathrm{F}\left(\mathrm{ab}^{\prime}\right)_{2}$ fragments and were obtained from Jackson ImmunoResearch Laboratories. Probed blots were imaged using West Pico Dura chemiluminescent reagent (Pierce) and X-OMAT AR X-ray film (Kodak). Pre-stained protein molecular weight markers (BioRad) were included during electrophoresis to allow determination of apparent molecular weights of detected antigens. All experiments were repeated at least twice with similar results.

\section{Authors' contributions}

PL conceived the project, performed most of the computational analysis, and drafted the complete manuscript; BJD conceived the project and carried out the western blot experiments; JRN and JJM performed the subcellular fractionation and related enzyme assays; LS contributed to the collection and annotations of rTS sequences.

\section{Additional material}

\section{Additional File 1}

Deduced rTS protein sequences based on existing DNA sequences and modifications made to existing $r T S$ protein sequences.

Click here for file

[http://www.biomedcentral.com/content/supplementary/1471-

2164-6-125-S1.doc]

\section{Acknowledgements}

The authors would like to thank Dr. John M. Graham, JG Research Consultancy, UK, for his suggestions and advice for the preparation of iodixanol gradients. This work is partially supported by a developmental fund from Roswell Park Cancer Institute (PL) and NIH grants CAI0I5I5 (PL), EB002II 6 (BJD), CA43500 (JJM), and CAI6056 (RPCl).

\section{References}

I. Babbitt PC, Hasson MS, Wedekind JE, Palmer DR, Barrett WC, Reed GH, Rayment I, Ringe D, Kenyon GL, Gerlt JA: The enolase superfamily: a general strategy for enzyme-catalyzed abstraction of the $\alpha$-protons of carboxylic acids. Biochemistry 1996, 35:16489-1650I.

2. Dolnick B]: Cloning and characterization of a naturally occurring antisense RNA to human thymidylate synthase mRNA. Nucleic Acids Res 1993, 21:1747-1752.

3. Dolnick BJ, Black AR, Winkler PM, Schindler K, Hsueh CT: rTS gene expression is associated with altered cell sensitivity to thymidylate synthase inhibitors. Adv Enzyme Regul 1996, 36:165-180.

4. Dolnick BJ, Black AR: Alternate splicing of the rTS gene product and its overexpression in a 5-fluorouracil-resistant cell line. Cancer Res 1996, 56:3207-3210. 
5. Dolnick BJ, Lu K, Yin MB, Rustum YM: Recent advances in the study of rTS proteins. rTS expression during growth and in response to thymidylate synthase inhibitors in human tumor cells. Adv Enzyme Regul 1997, 37:95-109.

6. Dolnick BJ, Angelino NJ, Dolnick R, Sufrin JR: A novel function for the rTS gene. Cancer Biol Ther 2003, 2:364-369.

7. Burge C, Karlin S: Prediction of complete gene structures in human genomic DNA. J Mol Biol 1997, 268:78-94.

8. Ford T, Graham J, Rickwood D: lodixanol: a nonionic iso-osmotic centrifugation medium for the formation of self-generated gradients. Anal Biochem 1994, 220:360-366

9. Graham J. Ford T, Rickwood D: The preparation of subcellular organelles from mouse liver in self-generated gradients of iodixanol 4. Anal Biochem 1994, 220:367-373.

10. Zhou J, Bai $Y$, Weiner H: Proteolysis prevents in vivo chimeric fusion protein import into yeast mitochondria. Cytosolic cleavage and subcellular distribution. J Biol Chem 1995 270:16689-16693.

II. Wagner C: Symposium on the subcellular compartmentation of folate metabolism. INutr 1996, I 26: I228S-I234S

12. Samsonoff WA, Reston J, McKee M, O'Connor B, Galivan J, Maley G, Maley $\mathrm{F}$ : Intracellular location of thymidylate synthase and its state of phosphorylation. I Biol Chem 1997, 272: I328I-I 3285

13. Dolnick RY, Wu Q, Angelino NJ, Stephanie LV, Chow K, Sufrin JR, Dolnick B]: Enhancement of 5-Fluorouracil Sensitivity by an rTS Signaling Mimic in H630 Colon Cancer Cell. Cancer Res 2005, 65:5917-5924

14. Wang $X Q$, Rothnagel JA: 5 '-untranslated regions with multiple upstream AUG codons can support low-level translation via leaky scanning and reinitiation 3. Nucleic Acids Res 2004, 32:|382-|39|

15. Kozak M: Pushing the limits of the scanning mechanism for initiation of translation. Gene 2002, 299:1-34.

16. Lukaszewicz M, M F, Jerouville B, Stas A, Boutry M: In vivo evaluation of the context sequence of the translation initiation codon in plants. Plant Sci 2000, 154:89-98.

17. Nakao M, Barrero RA, Mukai Y, Motono C, Suwa M, Nakai K: Largescale analysis of human alternative protein isoforms: pattern classification and correlation with subcellular localization signals I. Nucleic Acids Res 2005, 33:2355-2363.

18. Philippe $H$, Douady $C J$ : Horizontal gene transfer and phylogenetics. Curr Opin Microbiol 2003, 6:498-505.

19. Koonin EV, Makarova KS, Aravind L: Horizontal gene transfer in prokaryotes: quantification and classification. Annu Rev Microbiol 200I, 55:709-742.

20. Gogarten JP, Doolittle WF, Lawrence JG: Prokaryotic evolution in light of gene transfer. Mol Biol Evol 2002, 19:2226-2238.

21. Altschul SF, Madden TL, Schaffer AA, Zhang J, Zhang Z, Miller W, Lipman DJ: Gapped BLAST and PSI-BLAST: a new generation of protein database search programs. Nucleic Acids Res 1997 25:3389-3402.

22. Claros MG, Vincens P: Computational method to predict mitochondrially imported proteins and their targeting sequences. Eur J Biochem 1996, 24 I:779-786.

23. Emanuelsson $\mathrm{O}$, Nielsen $\mathrm{H}$, Brunak $\mathrm{S}$, von HG: Predicting subcellular localization of proteins based on their $\mathbf{N}$-terminal amino acid sequence. J Mol Biol 2000, 300:1005-1016.

24. Nakai K, Horton P: PSORT: a program for detecting sorting signals in proteins and predicting their subcellular localization. Trends Biochem Sci 1999, 24:34-36.

25. Guda C, Guda P, Fahy E, Subramaniam S: MITOPRED: a web server for the prediction of mitochondrial proteins. Nucleic Acids Res 2004, 32:W372-W374.

26. Guda C, Fahy E, Subramaniam S: MITOPRED: a genome-scale method for prediction of nucleus-encoded mitochondrial proteins. Bioinformatics 2004, 20: $1785-1794$.

27. McCloskey DE, McGuire IJ, Russell CA, Rowan BG, Bertino JR, Pizzorno G, Mini E: Decreased folylpolyglutamate synthetase activity as a mechanism of methotrexate resistance in CCRF-CEM human leukemia sublines. I Biol Chem I991, 266:6|8|-6|87.

28. McGuire J], Russell CA, Balinska M: Human cytosolic and mitochondrial folylpolyglutamate synthetase are electrophoretically distinct. Expression in antifolate-sensitive and -resistant human cell lines. J Biol Chem 2000, 275: I30I2-130|6.
29. Abmayr SMWJL: Preparation of nuclear and cytoplasmic extracts from mammalian cells. Current protocols in molecular biology 21993:12.11.11-12.11.19.

30. Storrie B, Madden EA: Isolation of subcellular organelles. Methods Enzymol 1990, 182:203-225.

3I. Schmidt E: Glutamate dehydrogenase - UV assay. In Methods in enzymatic analysis 2 nd edition. Edited by: Bergemeyer HU. New York, Academic Press, Inc; 1974:650-656

32. BOXSHADE server 2005 [http://bioweb.pasteur.fr/seqanal/inter faces/boxshade-simple.html]
Publish with Bio Med Central and every scientist can read your work free of charge

"BioMed Central will be the most significant development for disseminating the results of biomedical research in our lifetime. "

Sir Paul Nurse, Cancer Research UK

Your research papers will be:

- available free of charge to the entire biomedical community

- peer reviewed and published immediately upon acceptance

- cited in PubMed and archived on PubMed Central

- yours - you keep the copyright
BioMedcentral 